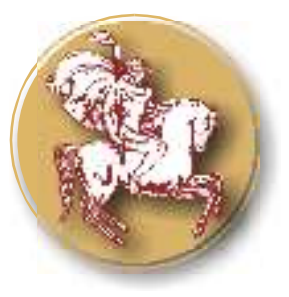

Trakia Journal of Sciences, No 4, pp 339-343, 2020

Copyright (C) 2020 Trakia University

Available online at:

http://www.uni-sz.bg

ISSN 1313-3551 (online)

doi:10.15547/tjs.2020.04.008

Original Contribution

\title{
MODEL OF INFLUENCE OF AT1, AT2 TYPE RECEPTORS ON SMOOTH MUSCLE CONTRACTIONS
}

\author{
K. Yankov*, G. Shivacheva \\ Faculty of Technics and Technologies, Trakia University, Stara Zagora, Branch Yambol, Bulgaria
}

\begin{abstract}
In the present work, the role of AT1 and AT2 receptors in the performance of angiotensin II-mediated processes has been analyzed. The involvement of each of the receptor types in regulation is determined by their separate exclusion from the feedback chain applying a suitable blocker. The resulting muscle contractions are modeled with a second-order differential equation. The transfer functions in the Laplace domain and state-space models of the contractions are derived from the models. The analysis of the models justifies the determination that AT1 type receptors participate in positive feedback and those of AT2 type in negative.
\end{abstract}

Key words: angiotensin II, feedback control, mathematical model, system identification

\section{INTRODUCTION}

The renin-angiotensin system (RAS) is an endocrine system that regulates waterelectrolyte balance and hemodynamics in the body. It affects processes such as inflammation, proliferation, cell growth, and carcinogenesis. The main effector of RAS is the octapeptide Angiotensin II (Ang II). It performs a number of functions: regulation of water-salt balance and electrolyte homeostasis, maintenance of vascular smooth muscle tone, etc. Ang II acts as an important regulator in the long-term maintenance of blood pressure and exerts a powerful contractile action on the gastrointestinal muscles. These multifaceted and significant functions of Ang II are accomplished by two separate angiotensin receptor types, designated AT1 and AT2 (1-3). They are feedback elements that guarantee the regulations in which Ang II is an agent. The study of the role of receptors in pharmacology and physiology is a prerequisite for determining the right therapy for many diseases.

\footnotetext{
*Correspondence to: Kaloyan Yankov, Faculty of Technics and Technologies, Trakia University, Branch Yambol, 38 Graf Ignatiev str., 8600 Yambol, Bulgaria. E-mail: kaloyan.yankov@trakiauni.bg,
}

The role of feedback in a regulatory process is usually assessed in the form of a transient process that is obtained by influence with an appropriate agent. This approach cannot be taken as accurate and unambiguous because it is not always accompanied by a comparison of numerical values. Another approach is to calculate time parameters using specialized software (4). In this way, the final result of the process is evaluated, but no answer is given, what regulatory mechanisms are being manifested.

The present work aims to evaluate the role of the two types of receptors involved in the implementation of Ang II-mediated processes using control theory methods. For this purpose, the transient processes obtained during the experiment will be identified by a second-order differential equation, and the role of the receptors will be evaluated by comparing the parameters of the identified models.

\section{MATHEMATICAL MODEL}

The subject of modeling is smooth muscle strips of the stomach. An input influence is Angiotensin II. Ang II acts on the smooth muscle of the stomach (SM), causing contraction. The output reaction is the strength of muscle contraction. The effect of two types 
of AT1 and AT2 receptors, which participate in feedback regulating the action of Ang II has been investigated (5).

\section{Data acquisition and processing}

The present work uses data provided by $\mathrm{P}$. Hadzhibozheva, Ph.D. (6). The experiment was performed on isolated, smooth muscle preparations from the stomach of healthy rats, Wistar line.

The role of each of the AT1 and AT2 receptors was examined by blocking them separately. For this purpose, a specific receptor antagonist is applied, after which the smooth muscle is treated with the contractile agent Ang II. The receptor blocking interrupts the action of the feedback loop in which it participates. The change in the force of contraction over time with the impact of the experimental agents was measured.
The following experiments were performed:

A) Administration of Ang II at a dose of 1 $\mu$ mol. Causes contraction involving AT1 and AT2 receptors. This experiment formed the control group.

B) Administration of PD 123319 at a dose of $100 \mathrm{nmol}$. This is a selective blocker for AT2 receptors. The resulting reaction is with the participation of the AT1 receptors in the feedback.

C) Administration of Losartan at a dose of 100 nmol. Selective blocker for AT1 receptors. The reaction involves AT2 receptors in the feedback.

D) The block diagram of the stomach muscle contractions with feedbacks is on Figure 1.

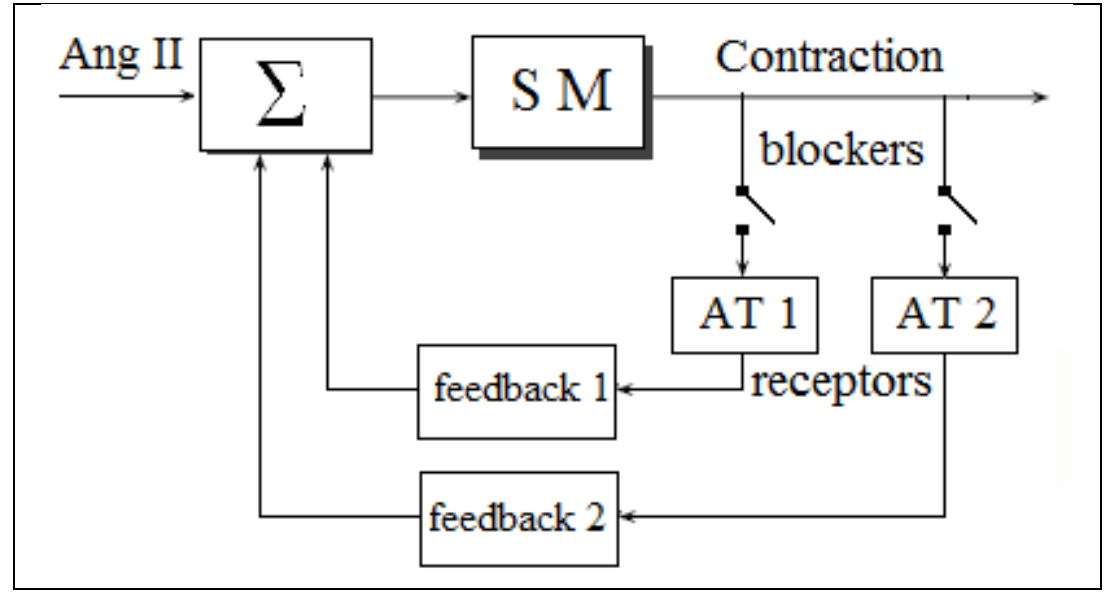

Figure 1. Block diagram with feedback control of Angiotensin II

The experiment was performed with the ISOSYS-Advanced 1.0 isolated system (Experimetria Ltd., Hungary). The primary processing of logged abbreviations: filtering, smoothing, scaling, graphical representation and storage of data for subsequent analysis were performed with specialized software Korelia-Processing (7). Figure 2 shows the spline interpolation of smooth muscle contractions for the three treatments described.

\section{Model identification}

A suitable mathematical model for identifying the three processes is a second-order differential equation (8):

$$
\frac{d^{2} y(t)}{d t^{2}}+2 \cdot \zeta \cdot \omega \cdot \frac{d y(t)}{d t}+\omega^{2} \cdot y(t)=K_{u} \cdot \omega^{2} \cdot U(t)
$$

where:

$\mathrm{y}(\mathrm{t})$ - the muscle contraction force;

$\mathrm{U}(\mathrm{t})$ - the input signal - Angiotensin II.

Parameters of the process:

$\mathrm{K}_{\mathrm{u}}$ - the sensibility of the system to the input influence;

$\omega^{2} \cdot K_{u}=G_{S S}$ - the steady state gain of the $\zeta$ - the damping ratio; system.

$\omega$ - the natural frequency of the system; 
These parameters are unknown and they must be calculated in order to identify each of the process.
The identification vector $\mathbf{Q}$ is:

$$
Q_{i}=\underset{i=A, B, C}{Q}\left(\zeta_{i}, \omega_{i}, K_{U i}\right)
$$

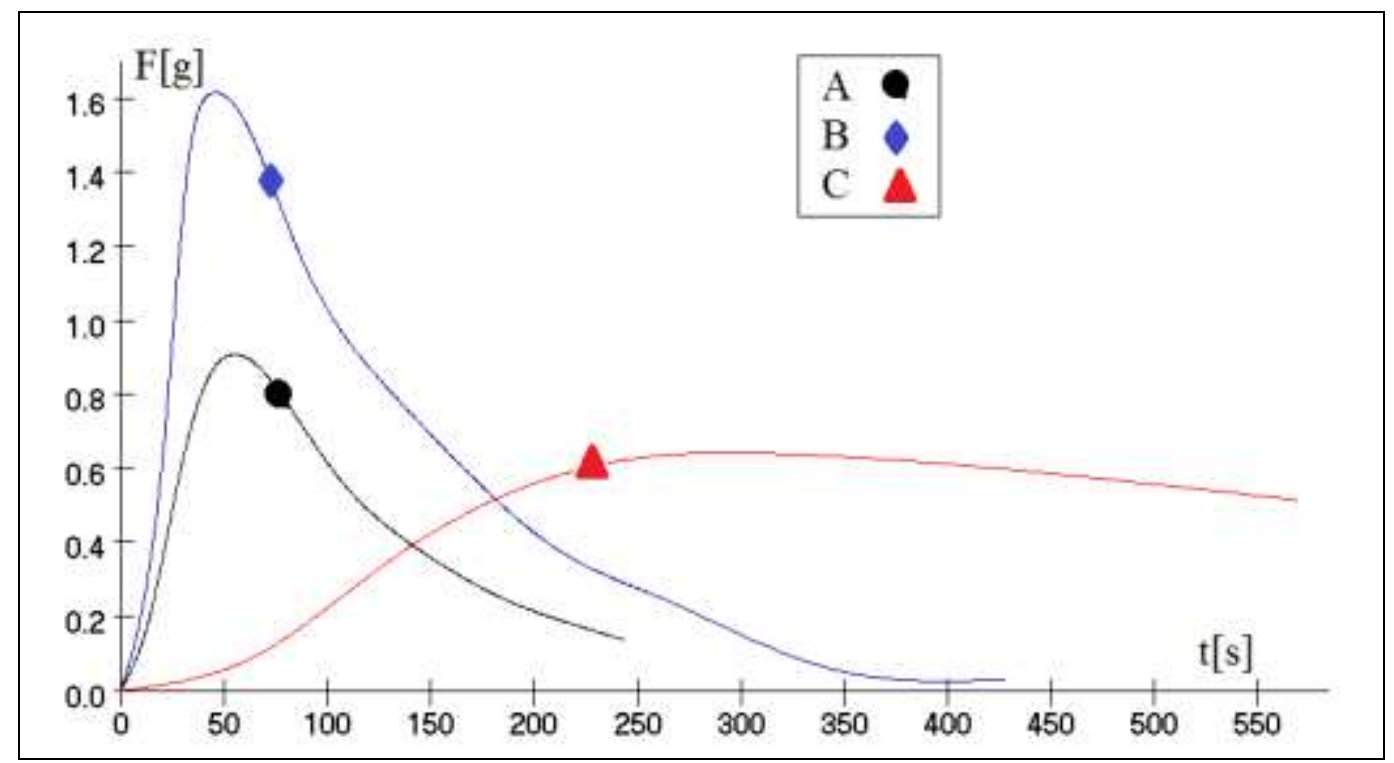

Figure 2. Spline interpolation of muscle contractions:
A) AT1 and AT2 type receptors.
B) AT1 type receptors.
C) AT2 type receptors.

The identification was made with the program Korelia-Ident (9). Fitting the model to the experimental data is done applying coordinate descent method. The criterion for the best approximation of the model to the data is the maximal value of the coefficient of determination $\boldsymbol{R}^{2}$. The identified data are in Table 1.

Table 1. Values of the identified parameters.

\begin{tabular}{|l|l|c|c|c|c|c|}
\hline \multirow{2}{*}{$\begin{array}{c}\text { Model of contractions } \\
\text { with receptors: }\end{array}$} & \multicolumn{4}{|c|}{ parameters } & \multirow{2}{*}{$\begin{array}{c}\text { Coeff } \\
\text { determ. } \boldsymbol{R}^{2}\end{array}$} \\
\cline { 3 - 7 } & $\boldsymbol{\zeta}$ & $\omega$ & $K_{u}$ & $\mathrm{G}_{\mathrm{SS}}$ & \\
\hline $\boldsymbol{A}$ & $\boldsymbol{A}) \boldsymbol{A T 1}$ AT2 receptors & 0.947 & 0.0196 & 38.02 & 0.0146 & 0.975 \\
\hline $\boldsymbol{B}$ & $\boldsymbol{B}) \boldsymbol{A T 1}$ receptors & 1.136 & 0.0199 & 77.09 & 0.0305 & 0.939 \\
\hline & C) AT2 receptors & 0.905 & 0.0032 & 168 & 0.0017 & 0.970 \\
\hline
\end{tabular}

\section{Transfer function}

A Transfer Function is the ratio of the output signal to the input signal, in the Laplace domain. A general second-order transfer function is:

$$
G(s)=\frac{G_{S S}}{s^{2}+2 \zeta \omega \cdot s+\omega^{2}}
$$

For all three cases, the transfer function is on Table 2.

Table 2. Transfer functions of the muscle contractions

\begin{tabular}{|c|c|c|c|}
\hline CASE & A) & B) & C) \\
\hline $\begin{array}{c}\text { transfer } \\
\text { function }\end{array}$ & 0.0146 & 0.0305 & $\frac{0.0017}{s^{2}+0.0371 . s+384 \mathrm{E}-6}$ \\
\hline
\end{tabular}

The transfer function can be exploited for computing the response to an arbitrary input signal.

\section{State-space representation}

State-space representation is required when creating a computer model of the process. The state variables of a system uniquely identify its current condition. State-space models provide 
a convenient and compact way to model and analyze high-order systems with multiple inputs and outputs.

The muscle contractions are described by the second-order equation, therefore two state variables $y_{1}(t)$ and $y_{2}(t)$, must be used to represent the model in state-space. Upon substitution:

$$
\mid \begin{aligned}
& y_{1}(t)=y(t) \\
& y_{2}(t)=\frac{d y(t)}{d t}
\end{aligned}
$$

In terms of the state variables, the original differential equation model (Eq.1) can be represented as:

$$
\left\|\begin{array}{c}
\frac{d y_{1}(t)}{d t} \\
\frac{d y_{2}(t)}{d t}
\end{array}\right\|=\left\|\begin{array}{cc}
0 & 1 \\
-\omega^{2} & -2 . \zeta . \omega
\end{array}\right\| \cdot\left\|\begin{array}{l}
y_{1}(t) \\
y_{2}(t)
\end{array}\right\|+\left\|\begin{array}{c}
0 \\
K_{u} \cdot \omega^{2}
\end{array}\right\| \cdot U(t)
$$

A matrix notation can be used to represent the system in a compact form:

$$
\dot{y}=A . y+B . u
$$

where:

y - state vector;

$\mathrm{u}$ - input vector;

A - state matrix. Represents the internal behavior of the system;
B - input matrix. Represents the relationship between state of the system and input influences.

Eigenvalues of the state matrix determine the type of stability at the critical points of a system. For all three cases of muscle contractions, state matrices $\mathbf{A}$ and its eigenvalues are in Table 3.

Table 3. State matrices and eigenvalues

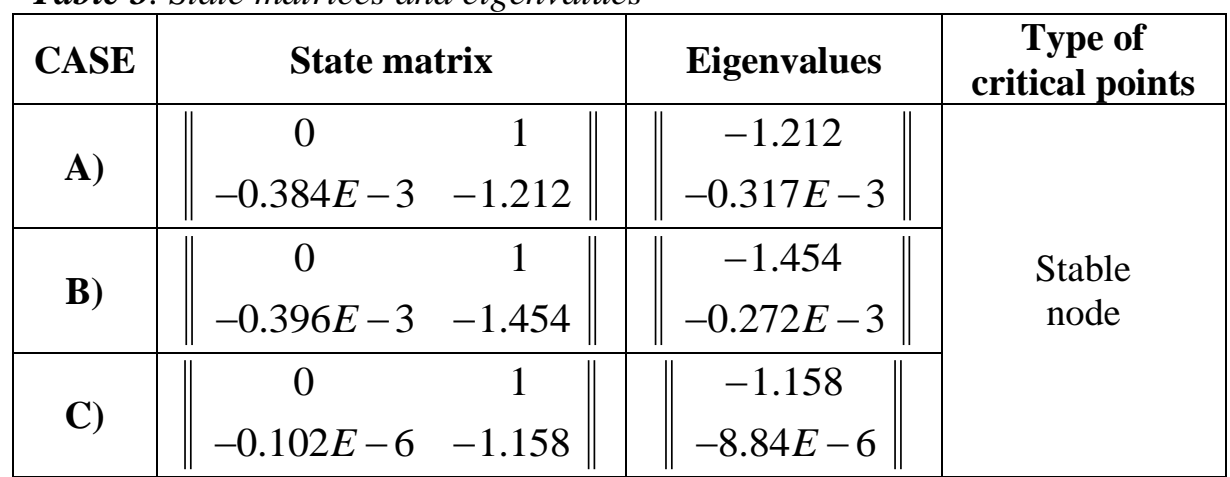

Negative real values of the eigenvalues define the system as stable, and the critical points - as a stable node.

\section{DISCUSSION}

For investigated muscle contractions, the damping ratios of the processes are approximately equal, fluctuating around 1 . This determines a fast process, without or with minimal overregulation. The fact that the damping ratio has slight differences in the three models indicates that the receptors tested have no effect on this parameter and it is a function of the system itself.

Feedback with AT2 type receptors only leads to a significant increase in the sensibility $\boldsymbol{K}_{\boldsymbol{u}}$ of the system. Activation of AT1 type receptors results in a fourfold decrease in this parameter.
The fact that the simultaneous involvement of the two receptors results in a lower $\boldsymbol{K}_{\boldsymbol{u}}$ relative to their self-influence suggests that these two types of receptors take part in an interaction that must be investigated.

The model shows that the AT1 receptors stabilize the natural frequency of the system. Their exclusion from regulation and participation of AT2 alone results in approximately a 6-fold reduction. The biological natural frequency is relevant to determining the optimal treatment period for drugs.

Increased steady-state gain $\boldsymbol{G}_{S S}$ is dominated by AT1 receptors - 15 times greater than AT2. This result suggests that the feedback with these receptors is positive. Positive feedback in 
physiology causes processes such as hysteretic switches and oscillations, and noise suppression. The fact that the two feedbacks are counteracting must find their physiological explanation. It can be assumed that other regulating mechanisms participate in the regulation of Ang II.

\section{CONCLUSION}

In this paper, the model of the influence of AT1, AT2 type receptors on smooth muscle contractions are studied. By applying the Ang II peptide and sequentially blocking both types of receptors, the contractile activity of the smooth muscle of the stomach was measured. The three processes are identified by the differential second-order equation. Based on the models, transfer functions and state-space models of the processes are derived.

Both types of receptors were found not to affect the damping ratio of the system. Type AT1 stabilizes the natural frequency since their exclusion from regulation leads to a 6 -fold decrease. Separately, both types of receptors lead to a higher sensibility of the system, but their joint involvement greatly reduces it. This fact implies interactions between them, which has not yet been studied.

The strong opposite dependence of the steadystate gain of the system on both receptors is a prerequisite for assuming that AT1 receptors participate in positive feedback and those of AT2 type in negative.

\section{ACKNOWLEDGMENTS}

This study was supported by Grant 2ФTT/31.03.2017, „Investigation of systems and processes by the phase plane method"from the Faculty of Technics and Technologies, Trakia University - Yambol, Bulgaria.

The authors express their gratitude to P. Hadzhibozheva, Ph.D., Trakia University, Faculty of Medicine, Bulgaria for the data provided.

\section{REFERENCES}

1. De Godoy M. A. and S. Rattan. Angiotensin-converting enzyme and angiotensin II receptor subtype 1 inhibitors restitute hypertensive internal anal sphincter in the spontaneously hypertensive rats. J Pharmacol Exp Ther, 2: 725-34, 2006.

2. Stegbauer, J. and T. M. Coffman. New insights into angiotensin receptor actions: from blood pressure to aging. Curr Opin Nephrol Hypertens, 1:84-88, 2011.

3. De Gasparo M, Catt KJ, Inagami T, Wright JW, Unger T. The angiotensin II receptors, Pharmacol Rev. 3:415-472, 2000.

4. Hadzhibozheva, P., A. Tolekova, Ts. Georgiev, G. Ilieva, R. Kalfin. Angiotensin II Receptors Type 2 and Gastrointestinal Tract Contractile Activity. Comptes rendus de l'Acadmie Bulgare des Sciences, 8:10911100, 2014.

5. De Godoy, M. A. and A. M. de Oliveira. Cross-talk between AT(1) and AT(2) angiotensin receptors in rat anococcygeus smooth muscle. J Pharmacol Exp Ther. 1:333-339, 2002.

6. Hadzhibozheva, P. Effects and mechanism of action of the peptide angiotensin II on visceral smooth muscles in norm and in experimental model of diabetes. Ph.D thesis. Institute of Neurobiology, Bulgarian Academy of sciences. 2016.

7. Yankov, K. Preprocessing of experimental data in Korelia software. Trakia Journal of Sciences, 3:41-48, 2010.

8. Yankov, K. System identification of biological processes, Proc. of the Int. Conf. Systems for Automation of Engineering and Research", Varna, Bulgaria, Sept. 2006, pp.144-149.

9. Yankov, K. Model Identification in Some Biochemical Analytical Tests. Applied Researches in Technics, Technologies and Education ARTTE, 3:267-276, 2014. 